\title{
Experiencia en el manejo de pacientes graves con COVID-19 en una unidad de terapia intensiva
}

\author{
Experience in the management of severe COVID-19 patients in an intensive care unit
}

\author{
J. Ojino Sosa-Garciaa ${ }^{1,5 *}$, Alan O. Gutiérrez-Villaseñor ${ }^{1,5}$, Alondra García-Briones ${ }^{2,5}$, \\ Juan P. Romero-González ${ }^{2,5}$, Eva Juárez-Hernández $z^{3,5}$ y Octavio González-Chon ${ }^{2,4,5}$ \\ ${ }^{1}$ Servicio de Medicina Interna y Medicina Crítica, Unidad de Terapia Intensiva, Hospital Médica Sur; ${ }^{2}$ Servicio de Anestesiología y Medicina Crítica, \\ Unidad de Terapia Intensiva, Hospital Médica Sur; ${ }^{3}$ Departamento de Investigación Clínica, Unidad de Investigación Traslacional, Hospital Médica \\ Sur; ${ }^{4}$ Departamento de Dirección, Hospital Médica Sur; ${ }^{5}$ Facultad de Medicina, Universidad Autónoma de México. Ciudad de México, México
}

\section{Resumen}

Objetivo: Describir las características clínicas y el manejo de pacientes graves con COVID-19. Método: Estudio observacional, descriptivo, longitudinal y restrospectivo. Resultados: Ingresaron 56 pacientes, el $80.3 \%(n=45)$ de sexo masculino, con un promedio de edad de 58 [46-67] años. Las principales condiciones de comorbilidad fueron obesidad, hipertensión y diabetes. El tiempo de inicio de los síntomas al ingreso fue de 9 [7-14] días, siendo los más frecuentes disnea, fiebre y tos seca. Los datos de laboratorio fueron linfopenia y elevación de deshidrogenasa láctica, fibrinógeno, dímero $D$, ferritina y proteína C reactiva. El $100 \%$ de los pacientes requirieron ventilación mecánica, con una mediana de tiempo de ventilación de 12 [6-17] días, y el 66\% $(n=37)$ requirieron posición en prono. El tratamiento farmacólogico fue a base de azitromicina, hidroxicloroquina, tocilizumab y esteroides, principalmente. Las complicaciones más frecuentes fueron lesión renal aguda, enfermedad tromboembólica venosa e infarto agudo al miocardio. La tasa de mortalidad fue del $17.8 \%(n=10)$. Conclusión: Los pacientes graves en nuestro hospital fueron en su mayoría personas de la tercera edad y con obesidad, siendo las variables de mayor puntaje SOFA y lesión renal aguda las asociadas con mayor mortalidad.

Palabras clave: Coronavirus. COVID-19. Manejo. Mortalidad. SARS-CoV-2.

\section{Abstract}

Objective: To describe the clinical characteristics and management of severe COVID-19 patients. Method: Observational, descriptive, longitudinal, and retrospective study. Results: 56 patients were admitted, of whom $80.3 \%(n=45)$ were males with a mean age of 58 years [46-67]. The main comorbidities were obesity, high blood pressure, and diabetes. Symptoms onset time at admittance to the ICU was 9 [7-14] days, of which the most frequent were dyspnea, fever, and dry cough. Laboratory data were lymphopenia; elevation of LDH, fibrinogen, D-dimer, ferritin and CRP. 100\% of the patients required mechanical ventilation, the median mechanical ventilation time was 12 [6-17] days, and $66 \%(n=37)$ required a prone position. The pharmacological treatment was mainly based on azithromycin, hydroxychloroquine, tocilizumab and steroids. The most frequent complications were acute kidney injury, venous thromboembolism and acute myocardial infarction. Mortality rate was

\footnotetext{
Correspondencia:

*J. Ojino Sosa-García

Puente de Piedra, 150

Col. Toriello Guerra, Del. Tlalpan

Fecha de recepción: 19-06-2020

C.P. 14050, Ciudad de México, México

E-mail: drintervista@gmail.com; jsosag@medicasur.org.mx

Fecha de aceptación: 29-06-2020

DOI: $10.24875 / C I R U .20000675$ 0009-7411/@ 2020 Academia Mexicana de Cirugía. Publicado por Permanyer. Este es un artículo open access bajo la licencia CC BY-NC-ND (http://creativecommons.org/licenses/by-nc-nd/4.0/).

Cir Cir. 2020;88(5):569-575 Contents available at PubMed www.cirugiaycirujanos.com
} 
Cirugía y Cirujanos. 2020;88(5)

$17.8 \%(n=10)$. Conclusion: The characteristics of the critically ill patients in our hospital were mostly elderly and obese, with the variables of higher SOFA score and acute kidney injury associated with higher mortality.

Key words: Coronavirus. COVID-19. Management. Mortality. SARS-CoV-2.

\section{Introducción}

A finales de diciembre de 2019 surgió la enfermedad por coronavirus 2019 (COVID-19) en Wuhan, provincia de Hubei, China, al reportar a la Organización Mundial de Salud 44 casos de neumonía de etiología no conocida, de los cuales 11 eran pacientes críticamente enfermos. Posteriormente se identificaría el virus causante, denominado SARS-CoV-2 con relación al síndrome respiratorio agudo grave, como causa de la enfermedad'.

De acuerdo con el mapa interactivo para el monitoreo de la enfermedad en el mundo, desarrollado por el Center for Systems Science and Engineering en la Johns Hopkins University en Baltimore ${ }^{2}$, hasta el día 5 de junio de 2020 se han reportado 6,724,516 casos confirmados, con 394,018 muertes por la enfermedad y 2,996,832 pacientes recuperados. Los países más afectados por la enfermedad hasta el momento son los Estados Unidos de América con 1,894,753 casos, Brasil con 614,941 casos, Rusia con 449,256 casos y el Reino Unido con 284,734 casos $^{3}$. En México se han reportado 110,026 casos, de los cuales han fallecido 13,170 pacientes ${ }^{4}$.

De acuerdo con lo publicado por Wang, et al. ${ }^{5}$, el porcentaje de pacientes que requieren ingreso en una unidad de terapia intensiva (UTI) es del $26.0 \%$, siendo las principales causas de admisión la presencia de disfunción orgánica manifestada por estado de choque, síndrome de insuficiencia respiratoria aguda, arritmias o falla cardiaca aguda.

El objetivo del presente estudio es describir las características clínicas y el manejo de los pacientes graves admitidos a la UTI con diagnóstico de COVID-19, en un hospital privado de tercer nivel de atención en la Ciudad de México, México.

\section{Método}

Es un estudio observacional, descriptivo, longitudinal y retrospectivo, llevado a cabo en la UTI del Hospital Médica Sur, en los meses de marzo a junio de 2020, previa autorización por el Comité de Ética en Investigación del Hospital (2020-EXT-461) y firma del consentimiento informado por el paciente o su representante legal.

Los criterios de inclusión fueron pacientes adultos de ambos sexos, mayores de 18 años, con deterioro progresivo de la función respiratoria manifestado por disnea e hipoxemia, con aumento de los requerimientos de aporte de oxígeno, y diagnóstico sospechoso o confirmado de COVID-19 según los datos clínicos y radiológicos compatibles con COVID-19 y con prueba reverse transcription polymerase chain reaction (RTPCR) SARS-CoV-2 negativa o RT-PCR SARS-CoV-2 de muestra nasofaríngea positiva, respectivamente. Se excluyó a las pacientes embarazadas.

Las variables evaluadas fueron la edad, el sexo, el índice de masa corporal, las características clínico-demográficas, los exámenes de laboratorio, los hallazgos radiográficos por tomografía computada de tórax, las escalas de pronóstico (Acute Physiology and Chronic Health Evaluation II [APACHE II], Sequential Organ Failure Assessment [SOFA] y Mexican SOFA [MEXSOFA]) a su ingreso, el manejo ventilatorio, el tratamiento farmacológico, las complicaciones y la mortalidad. Los datos fueron obtenidos de manera retrospectiva del expediente clínico al ingreso, y los paraclínicos y el tratamiento dirigido al ingreso y durante la hospitalización de los pacientes hasta su egreso por mejoría o defunción de la UTI o fecha de corte al día 5 de junio de 2020.

\section{Análisis estadístico}

La distribución de los datos se determinó mediante la prueba de Kolmogorov-Smirnov. Los datos categóricos se presentan en porcentaje e intervalos de confianza, y los datos continuos mediante medidas de tendencia central y dispersión. El análisis estadístico se realizó con el paquete estadístico SPSS v.20.

\section{Resultados}

Ingresaron 165 pacientes al Hospital Médica Sur, de los cuales el $34 \%(n=56)$ fueron admitidos a la UTI con diagnóstico confirmado por RT-PCR positiva para SARS-CoV-2 en el $96 \%$ de los casos $(n=54)$.

De los 56 pacientes admitidos a la UTI, el $80.3 \%$ $(n=45)$ eran varones, el promedio de edad era de 58 
Tabla 1. Características clínicas de los pacientes graves con COVID-19

\begin{tabular}{|c|c|c|c|}
\hline Caracaterísticas basales & Pacientes (\%) & Intervalo de confianza del $95 \%$ & Rango de referencia \\
\hline Sexo (masculino) & $45(80.3 \%)$ & $0.9-0.3$ & - \\
\hline Edad, años & $58[46-67]$ & - & - \\
\hline \multicolumn{4}{|l|}{ Comorbilidad } \\
\hline Obesidad & $28(50 \%)$ & $0.37-0.64$ & - \\
\hline Hipertensión & $20(35.7 \%)$ & $0.21-0.47$ & - \\
\hline Diabetes & $14(25 \%)$ & $0.12-0.35$ & - \\
\hline \multicolumn{4}{|l|}{ Manifestaciones clínicas } \\
\hline Días de inicio & $9[7-14]$ & - & - \\
\hline Disnea & $41(73.2 \%)$ & $0.62-0.86$ & - \\
\hline Fiebre & $39(69.6 \%)$ & $0.56-0.81$ & - \\
\hline Tos seca & $35(62.5 \%)$ & $0.50-0.76$ & - \\
\hline Mialgias & $25(44.6 \%)$ & $0.31-0.59$ & - \\
\hline Artralgias & $25(44.6 \%)$ & $0.31-0.59$ & - \\
\hline Dolor torácico & $10(17.8 \%)$ & $0.07-0.28$ & - \\
\hline Diarrea & $9(16 \%)$ & $0.06-0.26$ & - \\
\hline \multicolumn{4}{|l|}{ Hallazgos de laboratorio } \\
\hline Linfocitos & $900[500-1100]$ & - & $1.2-4 \times 10^{\wedge} 3 / \mu l$ \\
\hline Deshidrogenasa láctica & $343[285-486]$ & - & $98-192 \mathrm{U} / \mathrm{l}$ \\
\hline Dímero D & 1020 [600-3125] & - & $0-499 \mathrm{ng} / \mathrm{ml}$ \\
\hline Fibrinógeno & 699 [493-776] & - & $177-410 \mathrm{mg} / \mathrm{dl}$ \\
\hline Ferritina & 509 [132.7 - 1058.2] & - & $11-306.8 \mathrm{ng} / \mathrm{ml}$ \\
\hline Proteína C reactiva & $211[125.7-306.8]$ & - & $0-7.4 \mathrm{mg} / \mathrm{l}$ \\
\hline \multicolumn{4}{|l|}{ Hallazgos radiológicos } \\
\hline Vidrio despulido & 47 (83.9\%) & $0.75-0.95$ & - \\
\hline Consolidación & $39(69.6 \%)$ & $0.58-0.83$ & - \\
\hline \multicolumn{4}{|l|}{ Escalas de pronóstico } \\
\hline APACHE II & $14[12-19]$ & - & - \\
\hline SOFA & $7[6-9]$ & - & - \\
\hline MEXSOFA & $7[5-8]$ & - & - \\
\hline
\end{tabular}

[46-67] años y el índice de masa corporal era de 30.1 [27.3-32.4] kg/m². Las principales condiciones de comorbilidad fueron obesidad $(50 \%, \mathrm{n}=28)$, hipertensión $(30.3 \%, n=20)$ y diabetes $(25 \%, n=14)($ Tabla 1$)$.

La mediana del tiempo entre el inicio de las manifestaciones clínicas y el ingreso a la UTI fue de 9 [7-14] días, siendo los signos y síntomas más frecuentes disnea $(73.2 \%, n=41)$, fiebre $(69.6 \%, n=39)$, tos seca $(62.5 \%$, $\mathrm{n}=35)$, mialgias y artralgias $(44.6 \%, \mathrm{n}=25)$, dolor torácico $(17.8, n=10)$ y diarrea $(16 \%, n=9)$ (Tabla 1$)$.

Los hallazgos de laboratorio más frecuentes fueron linfopenia (900 [500-1100] × 10^3/ul) y elevación de la deshidrogenasa láctica (343 [285-486] U/I), del fibrinógeno (699 [493-776] mg/dl), del dímero D (1020 [600$3125] \mathrm{ng} / \mathrm{ml})$, de la ferritina (509 [132.7-1058.2] ng/ml) y de la proteína C reactiva (211 [125.7-306.8] mg/l) (Tabla 1). Los hallazgos radiológicos identificados por tomografía computada de tórax fueron imagen en vidrio despulido (83.9\%, $n=47)$ y áreas de consolidación $(69.6 \%, n=39)$ (Fig. 1).
Al momento del ingreso a la UTI se determinó el pronóstico según las diferentes escalas, siendo las medianas de estas: APACHE II 14 [12-19], SOFA 7 [6-9] y MEXSOFA 7 [5-8], con una probabilidad de mortalidad del $15 \%$, el $21.5 \%$ y el $20 \%$, respectivamente (Tabla 1).

El abordaje integral de los pacientes consistió en manejo avanzado de la vía aérea y ventilación mecánica invasiva con estrategias de protección pulmonar en el $100 \%$ de los casos. Las mediciones iniciales de los pacientes fueron: distensibilidad 37.4 [27-43] $\mathrm{ml} / \mathrm{cmH}_{2} \mathrm{O}$, presión de distensión pulmonar 14 [11-16] $\mathrm{cmH}_{2} \mathrm{O}$, presión meseta 27 [24-29] $\mathrm{cmH}_{2} \mathrm{O}$ y relación presión arterial de oxígeno/fracción inspirada de oxígeno 70 [50-100]. La modalidad de ventilación mecánica predominante fue controlada por volumen en el $82.1 \%(n=46)$, con una presión positiva al final de la espiración (PEEP) inicial de 12 [12-14] $\mathrm{cmH}_{2} \mathrm{O}$ y una fracción inspirada de oxígeno $\left(\mathrm{FiO}_{2}\right)$ del $73.3 \%$. La mediana en tiempo de ventilación 


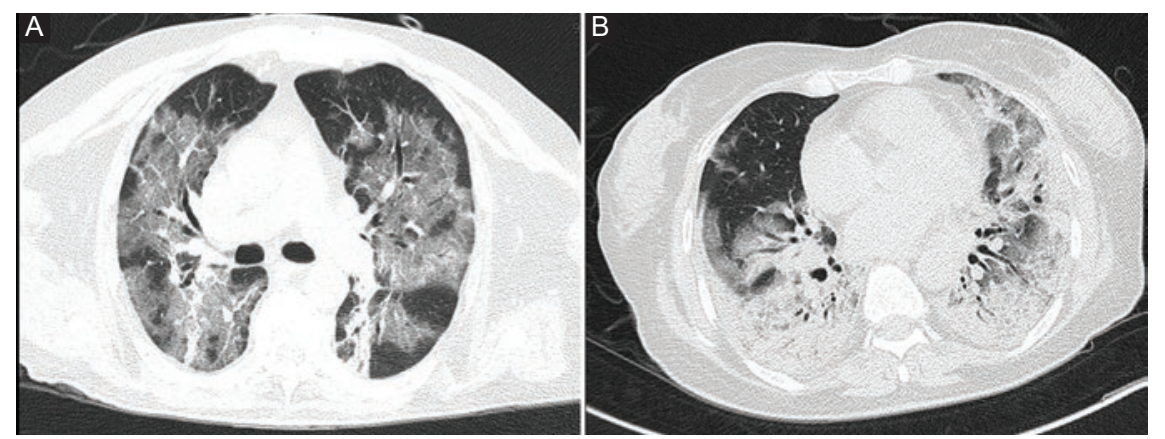

Figura 1. Hallazgos de la tomografía computada simple de tórax. A: imagen en vidrio despulido. B: imagen con áreas de consolidación bilateral.

Tabla 2. Parámetros de ventilación mecánica en los pacientes graves con COVID-19

\begin{tabular}{|c|c|c|}
\hline Parámetros & Valores & Intervalo de confianza del $95 \%$ \\
\hline Ventilación mecánica invasiva & $56(100 \%)$ & - \\
\hline \multicolumn{3}{|l|}{ Modalidad de ventilación } \\
\hline Controlada por volumen & $450[350-510] \mathrm{ml}$ & - \\
\hline PEEP & $12[12-14] \mathrm{cmH}_{2} \mathrm{O}$ & - \\
\hline $\mathrm{FiO}_{2}$ & $70[50-100] \%$ & - \\
\hline Posición en prono & $37(66 \%)$ & $0.52-0.78$ \\
\hline \multicolumn{3}{|l|}{ Medidas iniciales } \\
\hline Distensibilidad & $37[27-43] \mathrm{ml} / \mathrm{cmH}_{2} \mathrm{O}$ & - \\
\hline Presión de distensión pulmonar & $14[11-16] \mathrm{cmH}_{2} \mathrm{O}^{2}$ & - \\
\hline Presión meseta & $27[24-29] \mathrm{cmH}_{2}^{2} \mathrm{O}$ & - \\
\hline $\mathrm{PaO}_{2} / \mathrm{FiO}_{2}$ & $70[50-100]^{2}$ & - \\
\hline
\end{tabular}

Tabla 3. Desenlaces clínicos en los pacientes graves con COVID-19

\begin{tabular}{lc}
\hline Desenlaces & Pacientes (\%) \\
\hline Complicaciones & \\
Lesión renal aguda & $26(46.4 \%)$ \\
Enfermedad tromboembólica venosa & $6(10.7 \%)$ \\
Infarto agudo al miocardio & $2(3.5 \%)$ \\
Evento vascular cerebral isquémico & $1(1.8 \%)$ \\
Trombosis venosa cerebral & $1(1.8 \%)$ \\
Trombosis mesentérica & $1(1.8 \%)$ \\
Desenlaces clínicos & \\
Días de estancia en la UTI & $14[8-18]$ \\
Días de ventilación mecánica & $12[5-14]$ \\
Mortalidad & $10(17.8 \%)$ \\
\hline
\end{tabular}

mecánica fue de 12 [6-17] días y el $66 \%(n=37)$ requirió ventilación en posición en prono (Tabla 2).

El tratamiento farmacológico dirigido consistió en azitromicina $(75 \%, n=42)$, hidroxicloroquina $(67.8 \%, n=38)$, ritonavir $(39.2 \%, n=22)$, lopinavir $(30.3 \%, n=17)$, ivermectina $(17.8 \%, n=10)$, atazanavir $(10.7 \%, n=6)$, remdesivir $(3.5 \%, n=2)$ y oseltamivir $(10.7 \%, n=6)$. Dentro del manejo adjunto, se administró tocilizumab en el $53.5 \%$ $(n=30)$ y esteroides en el $46.4 \%(n=26)$.

Las complicaciones más frecuentes fueron lesión renal aguda $(46.4 \%, n=26)$, enfermedad tromboembólica venosa $(10.7 \%, n=6)$ e infarto agudo al miocardio $(3.5 \%, \mathrm{n}=2)$; hubo otras complicaciones menos frecuentes. De los pacientes que presentaron lesión renal aguda, el 17.8\% $(n=10)$ requirió manejo sustitutivo de la función renal (Tabla 3).

De los 56 pacientes que ingresaron a la UTI, cuatro continúan hospitalizados con soporte ventilatorio y 42 fueron retirados de la ventilación mecánica con aporte de oxígeno y egresaron a la sala de hospitalización específica de pacientes con COVID-19 para continuar su manejo convencional. De ellos, 39 pacientes han egresado del hospital por mejoría. La mediana de estancia en la UTI fue de 14 [8-18] días (Tabla 3).

La tasa de mortalidad en la UTI fue del $17.8 \%$ $(n=10)$, siendo las principales causas de defunción la tromboembolia pulmonar, el infarto agudo al miocardio, el evento vascular cerebral y la coagulación intravascular diseminada (Tabla 3). 
Tabla 4. Comparación de las variables según la mortalidad en pacientes sobrevivientes y no sobrevivientes

\begin{tabular}{|c|c|c|c|}
\hline Variable & Sobrevivientes ( $n=46$ ) & No sobrevivientes $(n=10)$ & $p$ \\
\hline \multicolumn{4}{|l|}{ Datos demográficos } \\
\hline Edad & $56[46-66]$ & $67[46-74]$ & 0.588 \\
\hline Sexo (hombre) & $77.8 \%(35)$ & $90 \%(9)$ & 0.667 \\
\hline Índice de masa corporal & $30.1[26.8-32.8]$ & $30[26.9-32.7]$ & 0.874 \\
\hline \multicolumn{4}{|l|}{ Comorbilidad } \\
\hline Obesidad & $51.1 \%(23)$ & $50 \%(5)$ & 1.000 \\
\hline Hipertensión & $31.1 \%(14)$ & $50 \%(5)$ & 0.288 \\
\hline Diabetes & $24.4 \%(11)$ & $20 \%(2)$ & 1.000 \\
\hline \multicolumn{4}{|l|}{ Manifestaciones clínicas } \\
\hline Inicio síntomas & $9[7-13]$ & $9[6-26]$ & 0.775 \\
\hline Disnea & $71 \%(32)$ & $90 \%(9)$ & 0.0423 \\
\hline Fiebre & $71 \%(32)$ & $60 \%(6)$ & 0.479 \\
\hline Tos seca & $66.7 \%(30)$ & $50 \%(5)$ & 0.469 \\
\hline \multicolumn{4}{|l|}{ Marcadores de inflamación } \\
\hline Linfopenia & $800[500-1075]$ & 1000 [700-1650] & 0.295 \\
\hline Deshidrogenasa láctica & $343[227-439]$ & 598.5 [336.5-962.5] & 0.650 \\
\hline Fibrinógeno & 699 [459-811] & 493 [493-699] & 0.250 \\
\hline Dímero D & $935[582-1490]$ & 3200 [1970-35200] & 0.083 \\
\hline Ferritina & $524.3[149.5-1026.4]$ & $452.7[0-1116]$ & 1.000 \\
\hline \multicolumn{4}{|l|}{ Escalas de pronóstico } \\
\hline APACHE II & $14[11.5-19]$ & $14.5[11.7-22.5]$ & 0.874 \\
\hline SOFA & $7[6-8]$ & $8[7-10.2]$ & 0.027 \\
\hline MEXSOFA & $7[5-8]$ & $7[4.7-8.0]$ & 0.866 \\
\hline \multicolumn{4}{|l|}{ Ventilación mecánica } \\
\hline Distensibilidad & $37[27.2-47.4]$ & $35.5[26.5-40.7]$ & 0.755 \\
\hline $\mathrm{PaO}_{2} / \mathrm{FiO}_{2}$ & $65[50-100]$ & $100[85-100]$ & 0.052 \\
\hline Posición prono & $66.6 \%(30)$ & $70 \%(7)$ & 1.000 \\
\hline Días de ventilación & $12[6-16]$ & $14[5-18]$ & 0.665 \\
\hline \multicolumn{4}{|l|}{ Tratamiento dirigido } \\
\hline Azitromicina & $73.3 \%(33)$ & $80 \%(8)$ & 1.000 \\
\hline Hidroxicloroquina & $68.9 \%(31)$ & $70 \%(7)$ & 1.000 \\
\hline Ritonavir & $40 \%(18)$ & $40 \%(4)$ & 1.000 \\
\hline Lopinavir & $28.9 \%(13)$ & $40 \%(4)$ & 0.479 \\
\hline Tocilizumab & $55.6 \%(25)$ & $40 \%(4)$ & 0.490 \\
\hline Esteroides & $57.8 \%(26)$ & $80 \%(8)$ & 0.287 \\
\hline \multicolumn{4}{|l|}{ Complicaciones } \\
\hline Lesión renal aguda & $40 \%(18)$ & $80 \%(8)$ & 0.035 \\
\hline Enfermedad tromboembólica venosa & $6.7 \%(3)$ & $30 \%(3)$ & 0.066 \\
\hline Infarto agudo al miocardio & $2.2 \%(1)$ & $10 \%(1)$ & 0.333 \\
\hline
\end{tabular}

En la comparación de las variables clínicas de acuerdo con la mortalidad, los pacientes no sobrevivientes mostraron un puntaje mayor de SOFA (7 [6-8] vs. 8 [7-10.2]; $p=0.027$ ) y un mayor porcentaje de lesión renal aguda $(40 \%[\mathrm{n}=18]$ vs. $80 \%[\mathrm{n}=8]$; $\mathrm{p}=0.035$ ) (Tabla 4).

\section{Discusión}

La población de América Latina presenta características epigenéticas distintas a las de otros continentes e incluso a la de los Estados Unidos de América y Canadá; sin embargo, el comportamiento de la COVID-19 no resulta ser distinto a lo descrito en la literatura. En México, dentro de las características epidemiológicas de la enfermedad predomina en el sexo masculino, con un promedio de edad de 45 años, y afecta principalmente a los mayores de 60 años; y las principales condiciones de comorbilidad son la hipertensión arterial, la diabetes, la obesidad, el tabaquismo, la insuficiencia renal crónica y la enfermedad pulmonar obstructiva crónica, entre otras. Por otra 
parte, los porcentajes de ocupación hospitalaria y de la UTI son del $44 \%$ y el $38 \%$ respectivamente, con una tasa de mortalidad general del $11.9 \%$.

Entre las características epidemiológicas de los pacientes graves que ingresaron a la UTI existe un predominio del sexo masculino, con una relación de 4:1, lo cual es similar a lo observado en el resto de la nación y en países como China, Italia y los Estados Unidos de América ${ }^{5-13}$. En cuanto a la edad, los pacientes graves fueron mayores de 60 años ${ }^{6,9-13}$ y presentaban comorbilidad, como obesidad, hipertensión y diabetes, mismos que han sido reportados en diferentes series de casos en todo el mundo ${ }^{5-13}$. Es importante mencionar que en esta población predomina la obesidad como factor de riesgo, lo cual se correlaciona con la alta prevalencia de esta condición en el país, que es del $32 \%$ en las personas mayores de 20 $a_{n}{ }^{14}$. Por otra parte, los primeros casos que ingresaron a la UTI fueron importados de Italia $(n=3)$, los Estados Unidos ( $n=3$ ), España $(n=1)$ y Turquía $(n=1)$, y el resto fue por transmisión comunitaria.

El periodo de inicio de las manifestaciones clínicas al ingreso a la UTI de la población estudiada fue de 9 días, similar a lo reportado en otras series de casos (7-10 días) $)^{8,11,13}$. Los signos y síntomas más frecuentes fueron disnea, fiebre y tos seca, similar a lo descrito en pacientes graves que requirieron ingresar a una UTI ${ }^{12,13}$.

Los hallazgos de laboratorio más comunes fueron linfopenia y elevación de la deshidrogenasa láctica y del dímero $\mathrm{D}$, de modo similar a lo descrito en la población general ${ }^{5,7-9,11-13}$, y elevación de la ferritina ${ }^{9,11}$ y de la proteína $C$ reactiva ${ }^{7,9}$. Otro dato característico de la población estudiada fue la elevación del fibrinógeno, que se asocia a mayor riesgo de eventos trombóticos en los pacientes con COVID-19. Dentro de los datos tomográficos, los principales fueron imagen en vidrio despulido y áreas de consolidación, al igual que en la población general y en pacientes graves $^{5,7,8,11-13}$.

En relación a las puntuaciones en las escalas de pronóstico al ingreso de los pacientes graves a la UTI, fueron de 14 para APACHE II y de 7 para SOFA, a diferencia de lo reportado por Wang, et al. ${ }^{5}$, en cuyo estudio fueron de 17 y 5 puntos, respectivamente; esta diferencia pudo deberse al grado de disfunción respiratoria de nuestra población.

El criterio de ingreso de los pacientes graves a la UTI fue deterioro progresivo de la función respiratoria manifestado por disnea e hipoxemia, con necesidad de manejo avanzado de la vía aérea y de ventilación mecánica invasiva, razón por la cual se abordó en el
$100 \%$ de los casos; a diferencia de otros estudios en los que se reporta una variabilidad del $47 \%$ al $88 \%$ de los casos, debido a que utilizaron otras estrategias de aporte de oxígeno, como la cánula nasal de alto flujo o la ventilación mecánica no invasiva ${ }^{5,10-12}$. El promedio de distensibilidad fue de $37 \mathrm{ml} / \mathrm{cmH}_{2} \mathrm{O}$ y la presión de distensión pulmonar fue de $14 \mathrm{cmH}_{2} \mathrm{O}$, las cuales fueron mayor y menor, respectivamente, que las reportadas por Bhatraju, et al. ${ }^{13}$. Dentro de los parámetros de ventilación mecánica iniciales, la PEEP fue de $12 \mathrm{cmH}_{2} \mathrm{O}$ y la $\mathrm{FiO}_{2}$ fue del $73.3 \%$, datos similares a los descritos por Grasselli, et al. ${ }^{10}$. La estrategia de ventilación en posición en prono se proporcionó en el $66 \%$ de los casos, mayor que lo reportado en otros estudios (27-50\%) $)^{10-12}$.

Las recomendaciones para el tratamiento farmacológico dirigido en los pacientes graves en la UTI se basaron en la guía clínica institucional, la cual fue desarrollada por expertos clínicos del hospital de acuerdo con la evidencia científica y la experiencia de los estudios reportados en otros países. El tratamiento inicial consistió en la administración conjunta de azitromicina/hidroxicloroquina ${ }^{15}$, lopinavir/ritona$v^{1}{ }^{16} \mathrm{y}$ tocilizumab ${ }^{17}$. El $46.4 \%$ de los pacientes recibieron esteroides, de modo similar a lo reportado en otras series ${ }^{7,11}$. Dentro de las opciones terapéuticas para el manejo se incluyó el uso compasivo de remdesivir ${ }^{18}$. Es importante mencionar que el tratamiento dirigido se ajustó continuamente según la generación de evidencia científica sobre la efectividad y la seguridad de las diferentes intervenciones; sin embargo, no se puede asegurar que los desenlaces clínicos se deban a su aplicación en nuestra población.

Dentro de las complicaciones de la población estudiada, presentaron lesión renal aguda el $46.4 \%$ de los pacientes, al igual que en otros estudios, como una de las principales complicaciones ${ }^{5,7,8,11,12}$. Por otra parte, nuestros pacientes presentaron enfermedad tromboembólica venosa e infarto agudo al miocardio como segunda y tercera complicaciones, de modo similar a lo reportado por Zhou, et al."1.

La tasa de mortalidad de los pacientes graves con COVID-19 en la UTI fue del $17.8 \%$, menor que la publicada en otras series de pacientes bajo las mismas características $(26-97 \%)^{9-13}$, siendo la presencia de mayor puntaje SOFA y de lesión renal aguda las variables con significancia estadística en los pacientes no sobrevivientes.

Las principales limitaciones del estudio son que es retrospectivo, unicéntrico y con un tamaño de muestra pequeño, por lo que los resultados de las medidas 
de correlación entre las diferentes intervenciones deberán tomarse con reserva hasta tener un mayor número de pacientes.

\section{Conclusiones}

Las características principales de los pacientes que requirieron ingreso a la UTI en nuestro centro hospitalario que en su mayoría fueron personas de la tercera edad, con obesidad y valores altos de los marcadores de inflamación y de trombosis. Destaca la presencia de un puntaje mayor de SOFA y de lesión renal aguda como variables más frecuentemente asociadas con la mortalidad. Si bien es cierto que en la actualidad no existe una sola intervención que haya demostrado efectividad y seguridad en la COVID-19, las estrategias de soporte multisistémico y de trabajo en equipo multidisciplinario impactan de manera positiva en los desenlaces clínicos de los pacientes graves.

\section{Conflicto de intereses}

Los autores declaran no presentar ningún conflicto de intereses.

\section{Responsabilidades éticas}

Protección de personas y animales. Los autores declaran que para esta investigación no se han realizado experimentos en seres humanos ni en animales.

Confidencialidad de los datos. Los autores declaran que han seguido los protocolos de su centro de trabajo sobre la publicación de datos de pacientes.

Derecho a la privacidad y consentimiento informado. Los autores declaran que en este artículo no aparecen datos de pacientes.

\section{Bibliografía}

1. WHO. Pneumonia of unknown cause - China. (Consultado el 10 de abril de 2020.) Disponible en: https://www.who.int/csr/don/05-january-2020pneumonia-of-unkown-cause-china/en/

2. Dong E, Du H, Gardner L. An interactive web-based dashboard to track COVID-19 in real time. Lancet Infect Dis. 2020;3099:19-20.

3. Coronavirus COVID-19 (2019-nCoV). (Consultado el 5 de junio de 2020.) Disponible en: https:/gisanddata.maps.arcgis.com/apps/opsdashboard/ index.html\#/bda7594740fd40299423467b48e9ecf6

4. COVID-D. Comunicado Técnico Diario COVID-19 MÉXICO. 2020;18-20.

5. Wang D, Hu B, Hu C, Zhu F, Liu X, Zhang J, et al. Clinical characteristics of 138 hospitalized patients with 2019 novel coronavirus-infected pneumonia in Wuhan, China. JAMA. 2020;323:1061-9.

6. Coronavirus. gob.mx. (Consultado el 2 de junio de 2020.) Disponible en: https://coronavirus.gob.mx/

7. Guan W, Ni Z, Hu Y, Liang W, Ou C, He J, et al. Clinical characteristics of coronavirus disease 2019 in China. N Engl J Med. 2020;382:1708-720.

8. Huang C, Wang Y, Li X, Ren L, Zhao J, Hu Y, et al. Clinical features of patients infected with 2019 novel coronavirus in Wuhan, China. Lancet. 2020;395:497-506.

9. Richardson S, Hirsch JS, Narasimhan M, Crawford JM, McGinn T, Davidson KW, et al. Presenting characteristics, comorbidities, and outcomes among 5700 patients hospitalized with COVID-19 in the New York City area. JAMA. 2020;323:2052-9.

10. Grasselli G, Zangrillo A, Zanella A, Antonelli M, Cabrini L, Castelli A, et al. Baseline characteristics and outcomes of 1591 patients infected with SARS-CoV-2 admitted to ICUs of the Lombardy Region, Italy. JAMA. 2020;323:1574-81.

11. Zhou F, Yu T, Du R, Fan G, Liu Y, Liu Z, et al. Clinical course and risk factors for mortality of adult inpatients with COVID-19 in Wuhan, China: a retrospective cohort study. Lancet. 2020;395:1054-62.

12. Arentz M, Yim E, Klaff L, Lokhandwala S, Riedo FX, Chong M, et al. Characteristics and outcomes of 21 critically ill patients with COVID-19 in Washington State. JAMA. 2020;4720:2019-21.

13. Bhatraju PK, Ghassemieh BJ, Nichols M, Kim R, Jerome KR, Nalla AK, et al. Covid-19 in critically ill patients in the Seattle Region - case series. N Engl J Med. 2020;382:2012-22.

14. Reding-Bernal $A$, Sánchez-Pedraza V, Moreno-Macías $H$, Sobrino-Cossio S, Tejero-Barrera ME, Burguete-García Al, et al. Heritability and genetic correlation between GERD symptoms severity, metabolic syndrome, and inflammation markers in families living in Mexico City. PLoS One. 2017;12:e0178815.

15. Gautret $P$, Lagier JC, Parola $P$, Hoang VT, Meddeb L, Mailhe M, et al. Hydroxychloroquine and azithromycin as a treatment of COVID-19: results of an open- label non-randomized clinical trial. Int J Antimicrob Agents. 2020 Mar 20:105949. doi: 10.1016/j.ijantimicag.2020.105949. Online ahead of print.

16. Cao B, Wang Y, Wen D, Liu W, Wang J, Fan G, et al. A trial of lopinavir-ritonavir in adults hospitalized with severe Covid-19. N Engl J Med. 2020;382:1787-99.

17. Xu X, Han M, Li T, Sun W, Wang D, Fu B, et al. Effective treatment of severe COVID-19 patients with tocilizumab. Proc Natl Acad Sci U S A. 2020;117:10970-5.

18. Grein J, Ohmagari N, Shin D, Diaz G, Asperges E, Castagna A, et al. Compassionate use of remdesivir for patients with severe COVID-19. N Engl J Med. 2020;382(24):2327-36. 